\title{
Mandibular Fibrosarcoma and Bile Duct Adenoma in a Pet Rabbit (Oryctolagus cuniculi): A Case Report
}

\author{
Inge Thas ${ }^{1}$, Gerry M. Dorrestein ${ }^{2}$, Nathalie Ariel Cohen-Solal ${ }^{3}$ \\ ${ }^{1}$ DAP Thas, Gent, Belgium \\ ${ }^{2}$ Pathology Laboratory NOIVBD, Veldhoven, The Netherlands \\ ${ }^{3}$ Imagerie Medical Veterinaire, Brussels, Belgium \\ Email: Inge@dapthas.net, info@noivbd.nl, cohen-solal@carlet.be
}

Received 18 February 2014; revised 14 March 2014; accepted 22 March 2014

Copyright (c) 2014 by authors and Scientific Research Publishing Inc.

This work is licensed under the Creative Commons Attribution International License (CC BY). http://creativecommons.org/licenses/by/4.0/

(c) (i) Open Access

\section{Abstract}

This report describes the clinical manifestations and pathological findings in a 4-year-old neutered male dwarf rabbit diagnosed with a mandibular fibrosarcoma. The rabbit was evaluated for a unilateral mandibular swelling and an acute onset of oral blood loss. Physical examination confirmed a marked facial asymmetry with a swollen painful left mandible. Radiographic projections of the skull revealed a left mandibular mass extending into the oral cavity. Tissue biopsies demonstrated severe bone destruction and a presumptive pathological diagnosis of a mandibular osteosarcoma were made. A helical CT (computed tomography) study of the skull confirmed the severe mandibular bone destruction and the oral extension of the mass. The patient was treated palliative with oral meloxicam and subcutaneous penicillin injections. Six months later, the rabbit died in a natural way. Postmortem examination led to final diagnosis of severe left facial malformation due to mandibular fibrosarcoma. A bile duct adenoma was found in the liver. To the author's knowledge, this is the first description of a spontaneous mandibular fibrosarcoma in a pet rabbit.

\section{Keywords}

Bile Duct Adenoma, Facial Swelling, Fibrosarcoma, Mandible, Rabbit

\section{Introduction}

Spontaneous oral neoplastic disease appears to be a rare finding in rabbits and only very few cases of spontane-

How to cite this paper: Thas, I., Dorrestein, G.M. and Cohen-Solal, N.A. (2014) Mandibular Fibrosarcoma and Bile Duct Adenoma in a Pet Rabbit (Oryctolagus cuniculi): A Case Report. Open Journal of Pathology, 4, 32-40. 
ous mandibular or maxillary neoplasia have been described in literature [1]. Only three spontaneous mandibular tumours including one cementoma, one chondrosarcoma and one ossifying fibroma have been reported in pet rabbits [2] [3]. This case demonstrates the importance of considering neoplasia in the differential diagnosis of mandibular swelling in rabbits.

\section{Case Report}

A four-year-old neutered male dwarf rabbit was presented with a one-month history of a left mandibular swelling. Before initial presentation, significant oral blood loss was noted. On presentation the rabbit was bright and alert and in good overall body condition (bodyweight $1.64 \mathrm{~kg}$ ). Abnormalities noticed during physical examination included a marked facial asymmetry with a swollen, painful left mandible. Regional lymph nodes appeared normal upon palpation. Previous treatment by the referring veterinarian with oral enrofloxacin and meloxicam did not produce any improvement.

The rabbit was sedated and multiple radiographic projections of the skull were taken. Latero-lateral, left and right lateral oblique and dorsoventral views of the skull revealed a left mandibular expansile mass of soft tissue density with extensive bone lysis and incisor and cheek teeth malocclusion (Figure 1).

At this time, mandibular abscess with osteomyelitis was the tentative diagnosis. One week later, the rabbit was scheduled for further diagnostic procedures under general anaesthesia. During this period, bodyweight had decreased to $1.52 \mathrm{~kg}$. Following blood sampling from the lateral saphenous vein for a complete blood count and serum biochemistry panel, the rabbit was placed in dorsal recumbence. A skin incision was made on the ventral part of the left mandible and the subcutaneous tissues were bluntly dissected until visibility of the mandibular mass was achieved. Upon incision, the mandibular swelling appeared to contain mainly blood clots and serosanguineous fluid. Loose dental and bone fragments and purulent debris were removed. More bone samples were obtained with a rongeur. Soft tissue samples were collected using a $6 \mathrm{~mm}$ biopsy punch. Upon digital palpation and oral inspection, the bone septum between the oral cavity and the mandibular mass appeared to be very thin and movable. Pus and bone samples were collected for culture and sensitivity and tooth, bone and soft tissue samples for pathological evaluation. Due to the presence of infection, the skin wound was left to heal by secondary intention.

Postoperative care included subcutaneous administration of carprofen and buprenorphine for pain control and prophylactic enrofloxacin. The rabbit recovered uneventfully from anaesthesia, was offered a soft, liquid herbivore assisted feeding formula by syringe 3 - 4 times a day and continued on subcutaneous injections of buprenorphine, carprofen and enrofloxacin. Three days later, the patient was sent home and maintained on oral meloxicam and enrofloxacin. Topical wound care included a mixture of Iso-Betadine gel and powdered sugar.

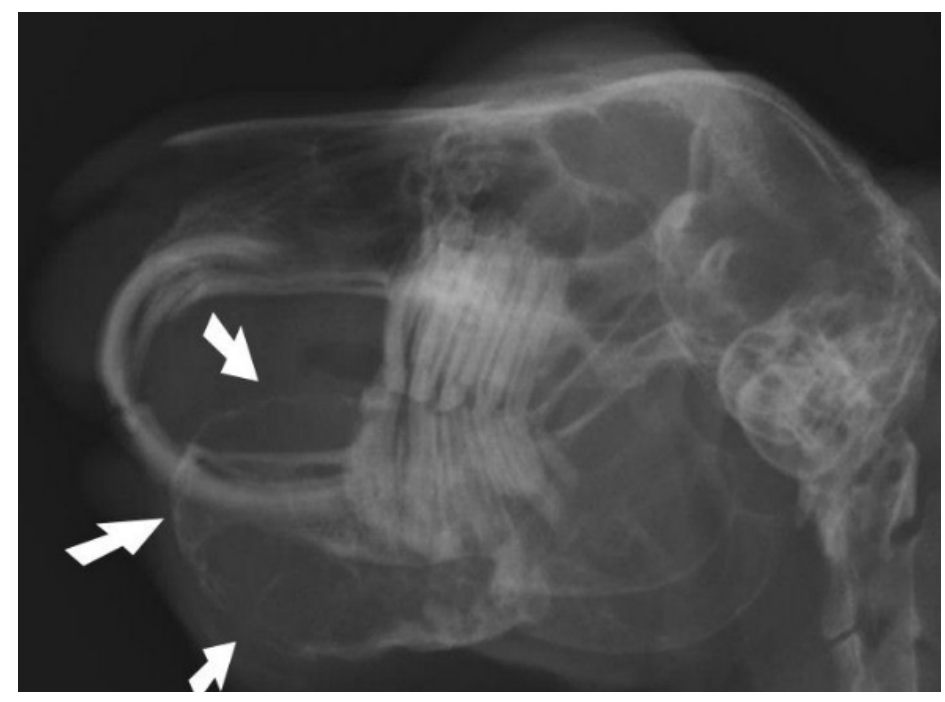

Figure 1. Latero-lateral view of the skull (slightly oblique). The mandibular mass is projecting into the oral cavity (arrows). Incisor and cheek teeth malocclusion is present. 
Blood haematology revealed heterophilia (80 per cent; reference range, 30 - 50 per cent), lymphocytopenia (18 per cent; reference range, 30 - 60 per cent) and decreased packed cell volume (PCV) (29.9 per cent; reference range, 33 - 48 per cent). Blood biochemistry revealed increased blood urea nitrogen (BUN) (13.49 mM; reference range, 6.14 - $8.38 \mathrm{mM}$ ), increased gamma glutamyltransferase (GGT) (13 iu/l; reference range, 0 - 7 $\mathrm{iu} / \mathrm{l}$ ), increased alkaline phosphatase (77 iu/l; reference range, 10 - $70 \mathrm{iu} / \mathrm{l}$ ) and decreased inorganic phosphate (1.2 mM; reference range, $1.28-1.92 \mathrm{mM}$ ) [4]. The rest of the biochemistry profile was unremarkable. Aerobic and anaerobic cultures of pus and bony fragments came back negative.

Soft tissue biopsies of the mandibular mass demonstrated extensive fibrous connective tissue with areas of squamous cell epithelium. In some of these epithelium islands, formation of new cartilaginous tissue was demonstrated. Some loose bone fragments were found in the fibrous tissue without reaction. One small cyst was demonstrated filled with mucous and in one area loose skeletal muscle transections were present in the connective tissue. No abnormalities were noted in the teeth fragments after decalcification. Because of the lack of cellular infectious reaction and the lack of tissue necrosis, a presumptive pathological diagnosis of a mandibular osteosarcoma was made.

Ten days later, the rabbit was presented for wound care including removal of abundant necrotic tissue through aggressive debridement. Oral meloxicam and enrofloxacin were continued.

Two weeks later a helical computed tomography (CT) scan of the head (1.9 mm of slice thickness, every 0.6 $\mathrm{mm}$, pitch 1) was performed under general anaesthesia to evaluate the extension of the mandibular mass. The CT images demonstrated an expansile lobulated soft tissue mass (45 Hounsfield units) arising from the left mandibular body and extending to the rostral part of the right mandibular body, displacing both mandibular incisors to the right. The mass was outlined by the interrupted cortical bone, showed extensive internal bony lyses with a loose reticular pattern and measured $36.8 \mathrm{~mm}$ in length, $25.4 \mathrm{~mm}$ in width and $25.1 \mathrm{~mm}$ in height. The medial part of the mass induced a mild pressure atrophy of the rostral part of the adjacent right mandible. The first and second left mandibular premolars were absent. The left mandibular ramus and three molars were intact (Figures 2-5).

A left hemi-mandibulectomy was proposed but declined by the owner. Three weeks later, the thin bone layer between the mandibular mass and the oral cavity had fractured. At this stage, euthanasia was proposed but de-

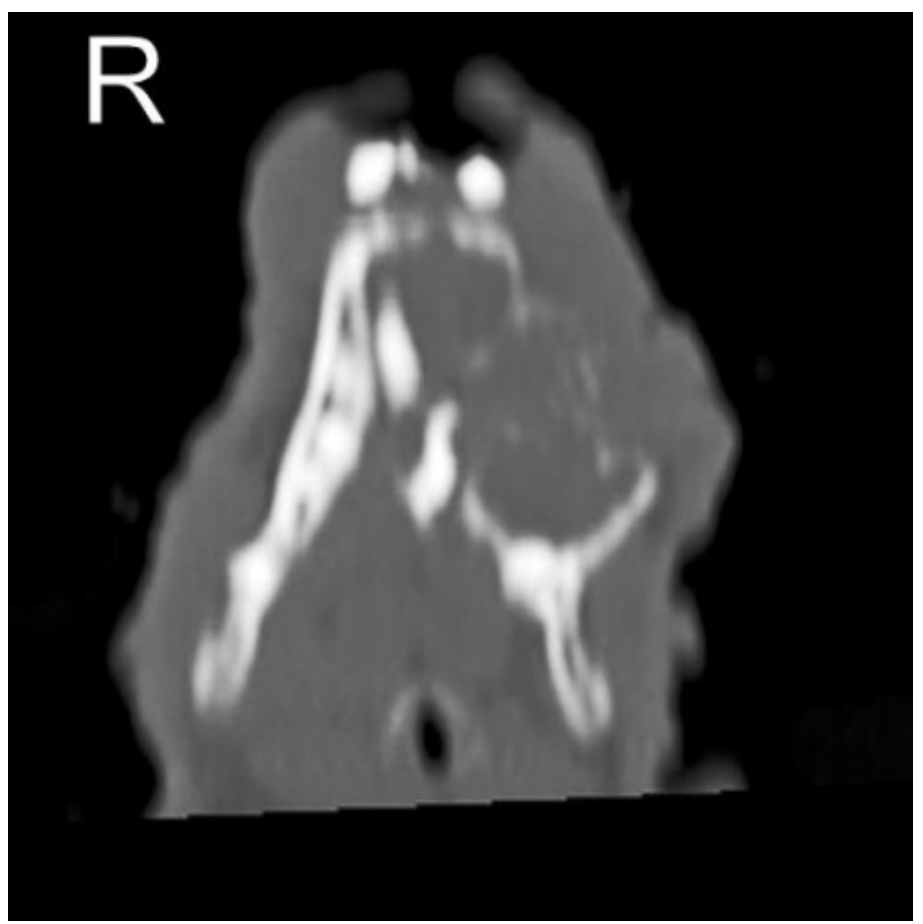

Figure 2. Latero-lateral view of the skull (slightly oblique). The mandibular mass is projecting into the oral cavity. Incisor and cheek teeth malocclusion is present. 


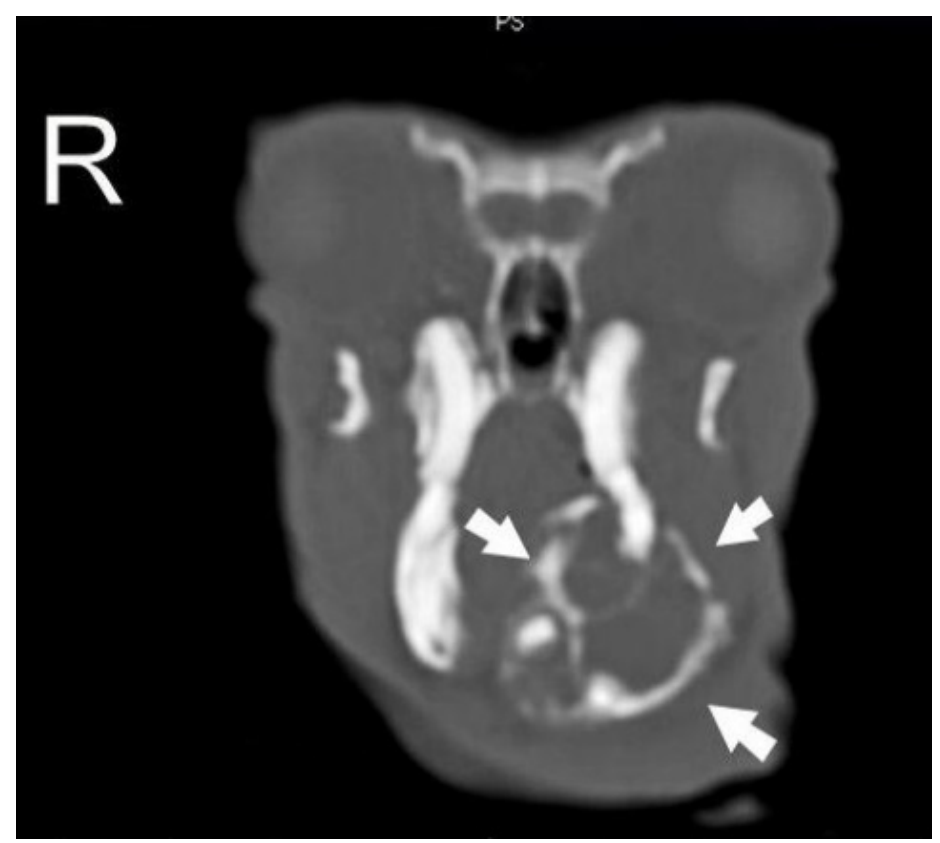

Figure 3. CT scan. Transverse view of the skull at the level of the zygomatic arch. The mandibular mass is clearly visible (arrows).

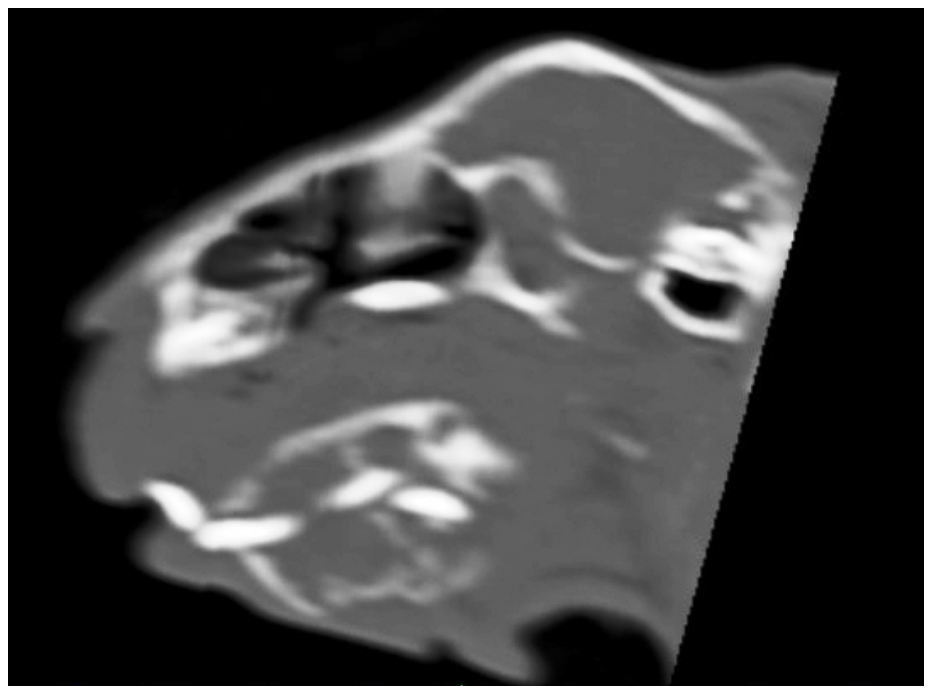

Figure 4. CT scan. Sagittal oblique view of the left mandibular body.

clined as well. The rabbit was continued on liquid herbivore assisted feeding formula and oral meloxicam for pain relief. Subcutaneous penicillin injections were given as a conservative palliative treatment.

The rabbit's appetite and bodyweight continued to decrease prior to dying five months later in a severely cachectic state (bodyweight $786 \mathrm{~g})$. Postmortem examination revealed a severe swollen mandible $(6 \times 5 \mathrm{~cm})$ and a small skin lesion on the left ventral mandible. The mandibular mass had an irregular surface with multiple soft areas. Mandibular incisor teeth were absent. A cross-cut section demonstrated a sponge-like structure with multiple partitions and cavities filled with necrotic tissue and haemorrhagic fluid (Figure 6).

The liver had multiple white focal spots up to $10 \mathrm{~mm}$ diameter (Figure 7).

The gastrointestinal system was filled with a pasty content. Microscopic examination of the gastro-intestinal contents was negative for parasites. And no normal faecal pellets were found.

Tissue samples of the tumour and different organs were fixed in 10 per cent buffered formalin, processed 


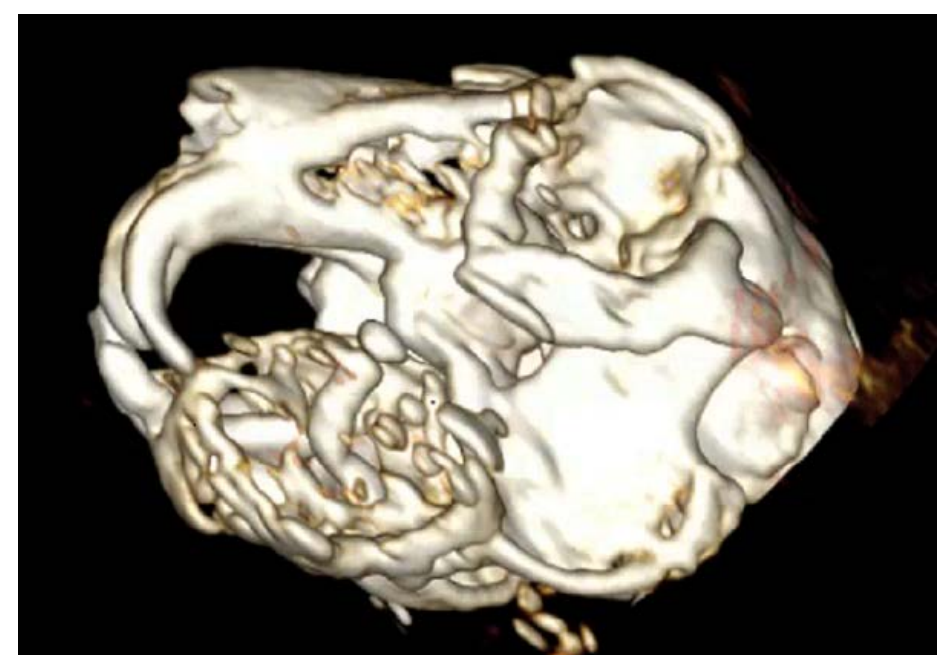

Figure 5. Volume rendering of the head. Left view of the skull. Severe bone destruction is obvious on the left mandible. The left mandibular incisor is not visible. The mandibular mass is extending into the oral cavity.

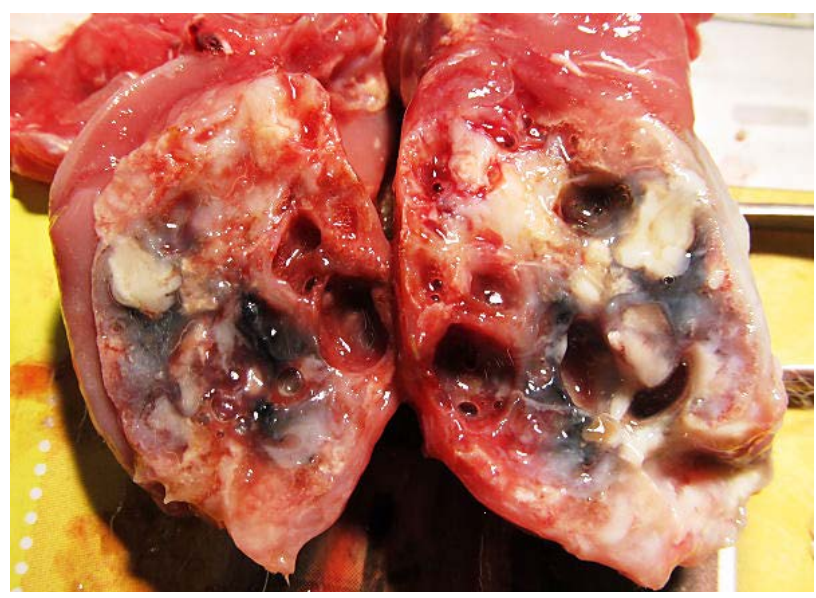

Figure 6. Postmortem examination. Transverse section through the left mandibular changes demonstrating severe bone destruction with formation of cavities.

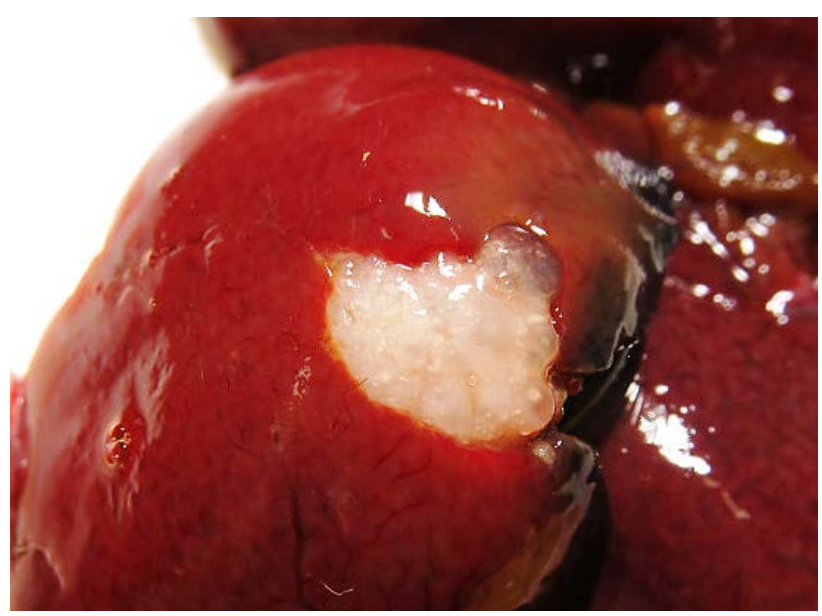

Figure 7. Postmortem examination. White focal spots in liver. 
routinely, sectioned (4 - $5 \mu \mathrm{m})$ and stained with haematoxylin and eosin (HE). Histopathological examination of the mandibular mass demonstrated abundant proliferation of fibrous tissue causing atrophy and destruction of pre-existing bone tissue (Figure 8).

Though severe bone destruction and malformation was present, no neoplastic changes were demonstrated in the bone. Some purulent material was present in the mandibular cavities. Necrosis was scarce. The mandibular soft tissue lesions were most consistent with fibrosarcoma. The liver was hyperaemic with obvious anisokaryosis and periportal areas demonstrating marked proliferation of fibrous tissue with minor inflammatory response. The white liver foci consisted of proliferating, primarily cubic (bile duct) epithelium with a lot of interstitial fibrous stroma. The sometimes cystic dilated cavities contained a thickened protein secrete (Figure 9).

Focal minor renal interstitial fibrosis was found and in those areas the renal tubules were dilated with protein-rich fluid. Minor pulmonary emphysema was present and the spleen was depleted of lymphocytes and contained mainly histiocytes and granulocytes.

Final diagnosis was severe malformation of the left mandible due to mandibular fibrosarcoma, and bile duct adenoma.

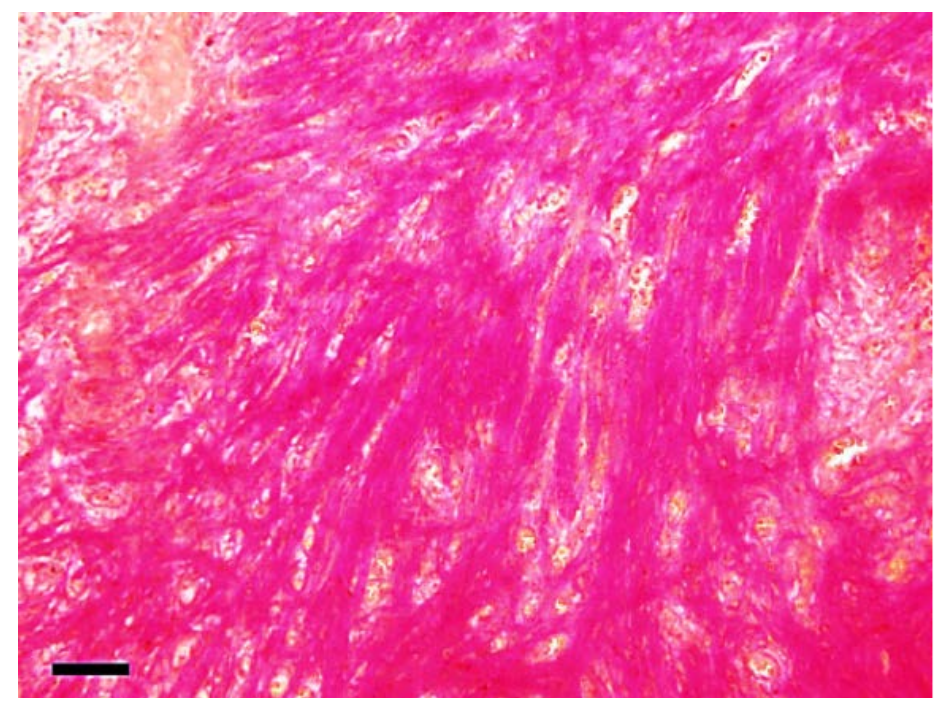

Figure 8. Mandibular proliferation showing connective tissue (van Gieson's stain, post-decalcification, bar $50 \mu$ ).

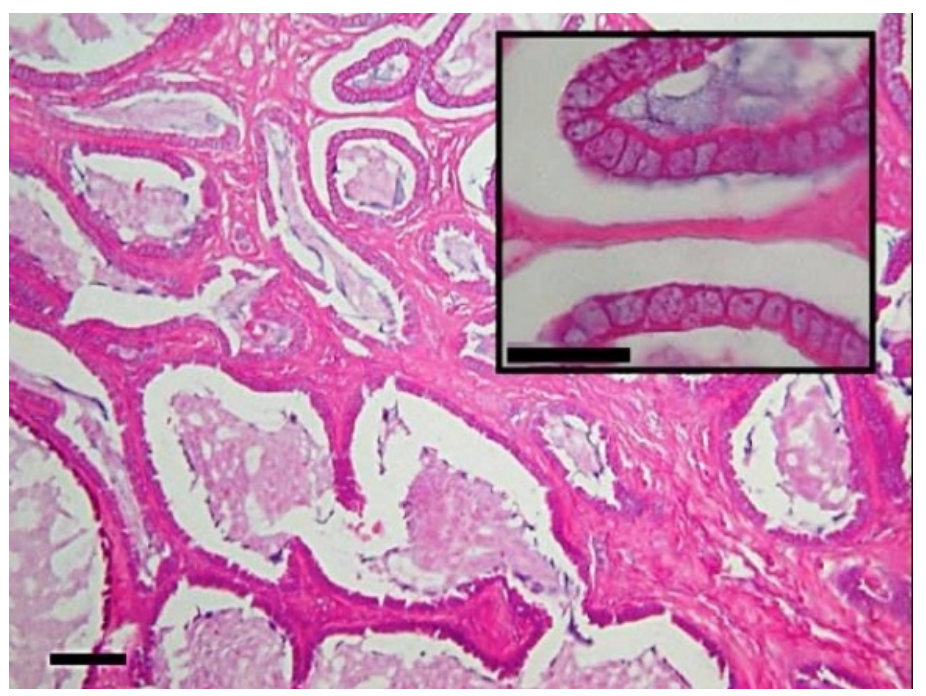

Figure 9. Liver with bile duct proliferation (HE $10 \times$, bar $50 \mu$ ). Insert (HE 40×, bar $20 \mu$ ). 


\section{Discussion}

Oral tumours are the fourth most common malignant neoplasia in dogs and cats and include odontogenic and non-odontogenic tumours. The majority of these are locally aggressive and slow to metastasize. Oral tumours can be staged into four different clinical stages using the TNM classification (primary tumour, regional lymph, node, distant metastasis). In most cases of oral neoplasia, the aetiology is unknown [5]. Osteosarcomas have been experimentally induced in rabbit mandibles with high-dose external-beam radiation or with intramedullary administration of carcinogen [6] [7]. Spontaneous oral neoplastic disease appears to be a rare finding in rabbits and rodents, only very few cases of spontaneous neoplastic mandibular or maxillary disease have been described in literature [1]. Spontaneous mandibular adamantinoma (one case), osteogenic fibroma (one case), osteogenic sarcoma (two cases), mucoepidermoid carcinoma (one case), fibrosarcoma (one case) and maxillary osteogenic sarcoma (one case) have been described in laboratory rabbits [8]-[14]. Only three spontaneous mandibular tumours including one cementoma, one chondrosarcoma and one ossifying fibroma have been reported in pet rabbits [2] [3].

Fibrosarcomas are malignant mesenchymal tumours derived from fibrous connective tissue and characterized by immature proliferating fibroblasts or undifferentiated anaplastic spindle cells. Oral fibrosarcoma is the third most common oral tumour in dogs and the second most common neoplasia in cats [5]. In dogs and cats, fibrosarcomas are locally invasive, and have a high recurrence rate after surgical excision, seldom metastasize and are most commonly found in the maxilla [5] [15]. Interscapular fibrosarcoma with similar histologic features to feline infection-site sarcoma was described in a dwarf rabbit after vaccination against rabbit myxoma and calicivirus [16]. Renfrew and others described an extraskeletal fibrosarcoma of the upper lip in a 7-year-old pet rabbit [17]. To the author's best knowledge, there is only one report in literature of a spontaneous oral fibrosarcoma in a laboratory New Zealand rabbit [14]. No cases have been reported in pet rabbits. The rabbit in this report had never been vaccinated and the cause of the mandibular fibrosarcoma remained unknown.

Clinical signs of oral fibrosarcoma in dogs and cats depend on the size and location of the mass and include facial asymmetry, difficulty apprehending food, secondary ulceration, halitosis, salivation caused by inability to swallow or associated pain, oral blood loss, weight loss and anorexia [5]. In the present case report, oral blood loss, painful facial mass, decreased appetite and weight loss were observed. Facial swelling was the main reason for initial presentation to the referring veterinarian.

The main differentials for a facial swelling in rabbits are abscess due to dental disease, bite wounds and/or foreign bodies, osteomyelitis, benign and malignant neoplasia and/or multicystic lesion [14] [18] [19]. In this report abscess, osteomyelitis and/or benign or malignant neoplasia were the initial differentials.

Diagnosis of oral fibrosarcoma in rabbits is based on histopathological examination. Staging of the neoplastic process should be performed in conjunction with the diagnostic evaluation. To rule out concurrent or metastatic disease, blood haematology, biochemistry and urine analysis should be performed. Imaging studies such as radiographs, CT, MRI can provide information on the extent of the neoplastic disease and absence or presence of metastasis [15]. Sedation or general anaesthesia is essential for proper positioning and for reducing breathing artefact. Because of the inability of standard radiography to demonstrate areas of bone loss and osteomyelitis due to superimposition of other bony and soft tissue structures and the superior contrast resolution with spiral CT scanners, CT of the head is extremely useful as an adjunct to traditional radiography and to determine margins and extent of tumour growth [14] [20]. In the present report, blood haematology and biochemistry were performed to rule out concurrent disease. Heterophilia and lymphopenia, as seen in this case, are common findings in stressed/diseased rabbits. Decreased appetite and dehydration may have caused elevated urea levels due to prerenal azotaemia. Liver GGT in rabbits is primarily present in bile duct epithelial cells and is therefore an indicator of hepatobiliary disease. In this rabbit, the increase in GGT was probably due to the bile duct adenoma [4].

Radiographic projections of the skull demonstrated extensive bone lyses and incisor and molar malocclusion. CT demonstrated the extension of the mass to the rostral part of the right mandibular body.

The recommended treatment in dogs and cats for oral fibrosarcoma includes aggressive surgery to obtain a 2 cm margin of tumour-free tissue, radiation therapy and/or chemotherapy [15] [21]. Hemi-mandibulectomy is a viable treatment modality in rabbits with oral tumours. Postoperative care including force feeding, analgesics and periodic dental trimming for malocclusion is essential [2]. Although in this case, the CT scan demonstrated initial involvement of the right mandible, left unilateral hemi-mandibulectomy was recommended but declined 
by the owner. Conservative treatment consisted of analgesic and antibiotic treatment to improve quality of life. Negative aerobic and anaerobic cultures may have been due to previous antibiotic treatment. The initial choice of antibiotic was enrofloxacin but because of severe bony involvement and the existence of a large connection with the oral cavity penicillin was initiated.

Spontaneous bile duct adenomas are a rare finding in pet rabbits and there are very few cases reported in literature. De Cubelis and others and Nasrin and others both reported a case of biliary cystadenoma of a 6-year-old pet female rabbit with a history of anorexia and an abdominal mass [22] [23]. The biliary adenoma found in the liver of this rabbit may have contributed to the final cachectic state but was probably an incidental finding.

In this rabbit, postmortem examination led to the final diagnosis of mandibular fibrosarcoma and bile duct adenoma. In the author's knowledge, this is the first report of a spontaneous mandibular fibrosarcoma with extensive bone destruction and cavity formation in a pet rabbit.

\section{Conclusions}

Mandibular neoplasia is an uncommon finding in pet rabbits. Diagnostic imaging and histopathology are the key diagnostic tests. The main differentials for a facial swelling in rabbits are abscesses due to dental disease, bite wounds and/or foreign bodies, osteomyelitis, benign and malignant neoplasia and/or multicystic lesion. In early stages, hemi-mandibulectomy is the treatment of choice. In more advanced cases, euthanasia should be considered.

Spontaneous bile ducts adenomas are infrequently reported in pet rabbits.

\section{References}

[1] Verstraete, F.J. (2003) Advances in Diagnosis and Treatment of Small Exotic Mammal Dental Disease. Seminars in Avian and Exotic Pet Medicine, 12, 37-48. http://dx.doi.org/10.1053/saep.2003.127877

[2] Miwa,Y. (2006) Mandibulectomy for Treatment of Oral Tumors (Cementoma and Chondrosarcoma) in Two Rabbits. Veterinary Journal, 8, 18-22.

[3] Whitten, K.A., Popielarczyk, M.M., Belote, D.A., McLeod, G.C. and Mense, M.G. (2006) Ossifying Fibroma in a Miniature Rex Rabbit (Oryctolagus cuniculus). Veterinary Pathology Online, 43, 62-64. http://dx.doi.org/10.1354/vp.43-1-62

[4] Harcourt-Brown, F. (2002) Clinical Pathology. In: Harcourt-Brown, F., Ed., Textbook of Rabbit Medicine, 2nd Edition, Butterworth-Heinemann, Edinburgh, 140-165.

[5] Dhaliwal, R.S. and Anthony, J.M.G. (2010) Tumors of the Mouth, Head and Neck. In: Ettinger, S.J. and Feldman, E.C., Eds., Textbook of Veterinary Internal Medicine, 7th Edition, Elsevier/Saunders, St. Louis, 1047-1053.

[6] Sato, K.I. and Horikoshi, T. (1975) Experimental Production of Osteogenic Sarcoma of the Mandible in Rabbits with 4-Nitroquinoline 1-Oxide. Unbound Medline, 66, 203-207.

[7] Buchholz, T.A., McCabe, K., Cobb, J., Haroff, H., Prestidge, B.R. and Ehler, W.J. (1999) TP53 Overexpression in Radiation-Induced Osteosarcoma of the Rabbit Mandible. Radiation Research, 151, 278-282. http://dx.doi.org/10.2307/3579939

[8] Brues, A.M. (1936) Osteogenic Sarcoma in a Rabbit. American Journal of Cancer, 28, 587-590. http://dx.doi.org/10.1158/ajc.1936.587

[9] Orr, J.W. (1936) Adamantinoma of the Jaw in a Rabbit. The Journal of Pathology and Bacteriology, 42, 703-704. http://dx.doi.org/10.1002/path.1700420316

[10] Weisbroth, S.H. and Hurvitz, A. (1969) Spontaneous Osteogenic Sarcoma in Oryctolagus cuniculus with Elevated Serum Alkaline Phosphatase. Laboratory Animal Care, 19, 263.

[11] Walberg, J.A. (1981) Osteogenic Sarcoma with Metastasis in a Rabbit (Oryctolagus cuniculus). Laboratory Animal Science, 31, 407.

[12] Hoover, J.P., Paulsen, D.B., Qualls, C.W. and Bahr, R.J. (1986) Osteogenic Sarcoma with Subcutaneous Involvement in a Rabbit. Journal of the American Veterinary Medical Association, 189, 1156.

[13] Gillett, C.S. and Gunther, R. (1990) Mandibular Mucoepidermoid Carcinoma in a Rabbit. Laboratory Animal Science, 40, 422-423.

[14] Brower, M., Goldstein, G.S., Ziegler, L.E. and Matise, I. (2006) Spontaneous Oral Fibrosarcoma in a New Zealand Rabbit. Journal of Veterinary Dentistry, 23, 96-99.

[15] Taney, K. and Smith, M.M. (2010) Oral and Salivary Gland Disorders. In: Ettinger, S.J. and Feldman, E.C., Eds., 
Textbook of Veterinary Internal Medicine, 7th Edition, Elsevier/Saunders, St. Louis, 1479-1486.

[16] Petterino, C., Modesto, P., Strata, D., Vascellari, M., Mutinelli, F., Ferrari, A. and Ratto, A. (2009) A Case of Interscapular Fibrosarcoma in a Dwarf Rabbit (Oryctolagus cuniculus). Journal of Veterinary Diagnostic Investigation, 21, 900-905. http://dx.doi.org/10.1177/104063870902100626

[17] Renfrew, H., Rest, J.R. and Holden, A.R. (2001) Extraskeletal Fibroblastic Osteosarcoma in a Rabbit (Oryctolagus cuniculus). Journal of Small Animal Practice, 42, 456-458. http://dx.doi.org/10.1111/j.1748-5827.2001.tb02502.x

[18] Gardner, D.G., Bunte, R.M., Sawyer, D.R. and Arlwohl, J. (1997) Multicystic Lesion of the Jaw in a Rabbit. Journal of the American Association for Laboratory Animal Science, 36, 78-80.

[19] Harcourt-Brown, F. (2002) Abscesses. In: Harcourt-Brown, F., Ed., Textbook of Rabbit Medicine, 2nd Edition, Butterworth-Heinemann, Edinburgh, 206-223.

[20] Capello,V. and Cauduro, A. (2008) Clinical Technique: Application of Computed Tomography for Diagnosis of Dental Disease in the Rabbit, Guinea Pig, and Chinchilla. Journal of Exotic Pet Medicine, 17, 93-101. http://dx.doi.org/10.1053/j.jepm.2008.03.006

[21] Hedlund, C.S. and Fossum, T.W. (2007) Surgery of the Digestive System. In: Fossum, T.W., Ed., Small Animal Surgery, 3rd Edition, Mosby/Elsevier, ST. Louis, 339-530.

[22] DeCubellis, J., Kruse, A.M., McCarthy, R.J., Zacher, L.A., Penninck, D., Watson, A.T., Parry, N., Donnelly, T.M. and Mayer, J. (2010) Biliary Cystadenoma in a Rabbit (Oryctolagus cuniculus). Journal of Exotic Pet Medicine, 19, 177-182. http://dx.doi.org/10.1053/j.jepm.2010.05.001

[23] Nasrin, A., Baharak, A. and Reza, K. (2012) Concurrent Cystic Endometrial Hyperplasia, Ovarian Luteoma and Biliary Cyst Adenoma in an Aged Rabbit (Oryctolagus cuniculus): Case Report and Literature Review. Asian Pacific Journal of Tropical Biomedicine, 2, 1975-1978. http://dx.doi.org/10.1053/j.jepm.2010.05.001 